\title{
Fast room-temperature functionalization of silicon nanoparticles using alkyl silanols $\dagger$
}

\author{
Alyssa F. J. van den Boom, (D) a Sidharam P. Pujari, (ID a Fatma Bannani, ${ }^{b}$ \\ Hafedh Driss (iD ${ }^{c}$ and Han Zuilhof (iD *acd
}

Received 3rd October 2019, Accepted 2nd January 2020

DOI: $10.1039 /$ c9fd00102f

Silicon nanoparticles (Si NPs) are a good alternative to conventional heavy metalcontaining quantum dots in many applications, due to their low toxicity, low cost, and the high natural abundance of the starting material. Recently, much synthetic progress has been made, and crystalline Si NPs can now be prepared in a matter of hours. However, the passivation of these particles is still a time-consuming and difficult process, usually requiring high temperatures and/or harsh reaction conditions. In this paper, we report an easy method for the room-temperature functionalization of hydrogen-terminated Si NPs. Using silanol compounds, a range of functionalized Si NPs could be produced in only $1 \mathrm{~h}$ reaction time at room temperature. The coated NPs were fully characterized to determine the efficiency of binding and the effects of coating on the optical properties of the NPs. It was found that Si NPs were effectively functionalized, and that coated NPs could be extracted from the reaction mixture in a straightforward manner. The silanol coating increases the quantum yield of fluorescence, decreases the spectral width and causes a small $(\sim 50 \mathrm{~nm})$ blue-shift in both the excitation and emission spectra of the Si NPs, compared to unfunctionalized particles.

\section{Introduction}

Semiconductor nanoparticles have proven to be useful for many different applications, including bio-imaging, ${ }^{1-3}$ LEDs, ${ }^{4-6}$ and solar cells. ${ }^{7-12}$ Silicon nanoparticles (Si NPs) are, in this regard, particularly interesting, due to their low toxicity and the high natural abundance of the starting material. ${ }^{13,14}$ However, several issues have so far stood in the way of their widespread use. First, synthesis

\footnotetext{
${ }^{a}$ Laboratory of Organic Chemistry, Wageningen University, Stippeneng 4, 6708 WE Wageningen, The Netherlands. E-mail: han.zuilhof@wur.nl

${ }^{b}$ Department of Chemistry, King Abdulaziz University, Jeddah, Saudi Arabia

${ }^{c}$ Chemical and Materials Engineering, Faculty of Science, King Abdulaziz University, Jeddah, Saudi Arabia

${ }^{d}$ School of Pharmaceutical Science and Technology, Tianjin University, 92 Weijin Road, Tianjin, P. R. China

$\dagger$ Electronic supplementary information (ESI) available. See DOI: 10.1039/c9fd00102f
} 
of the NPs is time-consuming and difficult, often requiring high temperatures or harsh chemicals. ${ }^{15-25}$ Second, once the Si NPs are formed, they have to be rapidly passivated to prevent the loss of their original properties. ${ }^{26}$ This is especially the case for hydrogen-terminated Si NPs (H-Si NPs). Such particles are of significant interest due to their near-ideal electronic and electrical properties, yet their susceptibility to oxidation requires extensive protection. In the literature, passivation of $\mathrm{H}$-Si NPs has been achieved by heating to $190^{\circ} \mathrm{C}$ in the presence of a suitable ligand, ${ }^{27}$ etching using phosphorous pentachloride followed by modification with an amine, ${ }^{20,28}$ thermally-promoted thiolation reactions, ${ }^{29}$ reacting with bifunctional alkenes ${ }^{26}$ halogenation followed by a Grignard reaction, ${ }^{24,30}$ platinum-catalyzed reactions, ${ }^{23,25,31}$ or reactions in the presence of radical initiators. $^{32,33}$ These procedures generally suffer from long reaction times and/or the use of non-trivial reaction conditions, making them less attractive for application on a larger scale. Furthermore, the type of ligand that can be attached in such procedures is usually limited by the reaction conditions used. In most cases, the ligand may also contain only one functional group, as having multiple functional groups interferes with the reaction. This limits the possibility for further functionalization. Clearly, an easy, fast, and versatile passivation method for H-Si NPs is still needed.

In this paper, we report the use of silanol compounds with a range of functional groups to passivate H-Si NPs (obtained via a microwave-based procedure developed in our group, ${ }^{34}$ building upon earlier protocols from He's group for other types of Si NPs). ${ }^{35,36}$ This silanol reaction showed promise due to the discovery that flat Si surfaces could readily be functionalized in this manner, ${ }^{37}$ yielding densely packed, hydrolytically stable monolayers in $<1 \mathrm{~h}$ at room temperature. As this reaction proceeded without the need for additional solvents or catalysts, this constitutes a significant advantage over previously mentioned routes. Here, only mono-hydroxylated silanols were considered, as silanols with two or three hydroxyl groups might yield multilayers on the surface of the Si NPs, as reported for flat surfaces. ${ }^{37}$ We show that passivation with silanols is an easy and fast method to obtain functionalized Si NPs. The silanol coating can easily be tuned by selecting silanols with different side groups, and due to the specificity of the reaction, even silanols with multiple functional groups can be used. Finally, we show it is possible to obtain pure, passivated Si NPs with good optical properties in less than $4 \mathrm{~h}$ of combined reaction $(1 \mathrm{~h})$ and purification $(3 \mathrm{~h})$ time.

\section{Experimental}

\section{Synthetic procedures}

Synthesis of silanol compounds. Silanol compounds were synthesized from their corresponding chlorosilanes using an adapted literature procedure (Scheme 1). ${ }^{37}$ In brief, an alkyldimethylchlorosilane (1 eq.) was added to an ice-cold mixture of triethylamine (1.5 eq.), water (4 eq.), and diethyl ether. The mixture was stirred for $1 \mathrm{~h}$ at room temperature, after which the liquid phase was isolated. Hexane was added, and the diethyl ether was evaporated in vacuo. The reaction mixture was cooled on an ice bath, and washed with ice-cold $0.5 \mathrm{M}$ aqueous $\mathrm{HCl}$. The organic phase was dried using magnesium sulfate, and the solvent was evaporated in vacuo to obtain the silanol. Full characterization of the silanols used in this study can be found in the ESI. $\dagger$ 
A

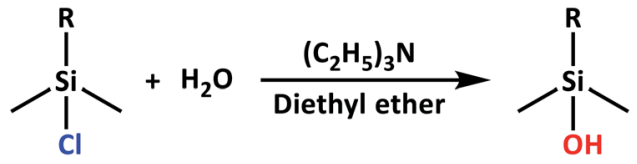

B

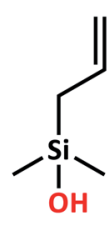

1

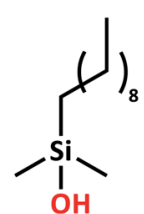

2

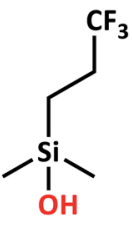

3

Scheme 1 (A) Reaction scheme for silanol synthesis. (B) Overview of silanols used in this study.

Synthesis of hydrogen-terminated silicon nanoparticles. H-Si NPs were prepared according to the protocol developed by Pujari et al. ${ }^{34}$ In brief: inside a glovebox $\left(\mathrm{O}_{2}<0.1 \mathrm{ppm}, \mathrm{H}_{2} \mathrm{O}<0.1 \mathrm{ppm}\right), 20 \mathrm{mg}(0.10 \mathrm{mmol})$ of sodium ascorbate and $0.8 \mathrm{ml}$ of DMSO were added to a microwave tube equipped with a stirrer bar. $0.2 \mathrm{ml}(1.08 \mathrm{mmol})$ of triethoxysilane was added, after which the tube was quickly sealed and transported to the microwave. The mixture was allowed to react for $10 \mathrm{~min}$ at $170{ }^{\circ} \mathrm{C}$ while stirring at $600 \mathrm{rpm}$. After the reaction, the tube was allowed to cool down and was transported back into the glovebox. There, it was opened, and the mixture was filtered with a $0.2 \mu \mathrm{m}$ PTFE syringe filter. The filtered brown solution was directly used for passivation.

Functionalization of silicon nanoparticles. Inside a glovebox, $0.2 \mathrm{ml}$ of the selected silanol is added to the filtered nanoparticle dispersion, and the resulting mixture is stirred for $1 \mathrm{~h}$ at room temperature. After this passivation step, the reaction mixture is taken out of the glovebox, and the DMSO is evaporated in vacuo $\left(70{ }^{\circ} \mathrm{C}, 1 \mathrm{mbar}\right)$; excess silanol is subsequently extracted using diethyl ether. After drying, the resulting dry brown flakes are finely ground with $15 \mathrm{ml}$ of toluene, added in several small fractions, to extract the functionalized NPs; large particles are allowed to sediment after the addition of each fraction, and the top layer of toluene is pipetted off. The combined toluene phases are dried prior to analysis. In a typical experiment, 20-35 $\mathrm{mg}$ of functionalized Si NPs were obtained in this way, depending on the ligand and the number of toluene fractions used.

Preparation of water-soluble particles. To prepare water-soluble Si NPs, a thiol sugar was attached to the allyldimethylsilanol-functionalized Si NPs via a thiol-ene click reaction. To a purified sample of $30 \mathrm{mg}$ of the functionalized Si NPs, a solution of $0.36 \mathrm{~g}(1.0 \mathrm{mmol})$ 1-thio- $\beta$-D-glucose tetraacetate and $0.05 \mathrm{~g}(0.2 \mathrm{mmol}) 2,2-$ dimethoxy-2-phenylacetophenone (DMPA) in 1:1 chlorobenzene and DMF was added inside a glovebox. ${ }^{38,39}$ The reaction mixture was stirred at room temperature for $3.5 \mathrm{~h}$ with $365 \mathrm{~nm}$ light irradiation. The distance between the lamp and the solution was $\sim 4 \mathrm{~cm}$, so as not to heat the sample. The lamp was removed, and the solvents were evaporated in vacuo. A light brown gel formed, from which Si NPs and DMPA were extracted using chlorobenzene. The solvent was again removed in vacuo, and the functionalized Si NPs were extracted using diethyl ether. 
To de-protect the acetate-capped sugar, the Si NPs were dispersed in a $0.015 \mathrm{M}$ solution of sodium methoxide in methanol. ${ }^{40}$ The reaction mixture was stirred for $45 \mathrm{~min}$, and sodium methoxide was precipitated out of the solution by the addition of anhydrous pentane. The solvents in the clear dispersion were removed in vacuo to obtain $27 \mathrm{mg}$ of dry thioglucose-coated Si NPs for analysis.

\section{Materials and methods}

NMR measurements. NMR measurements were conducted on a $400 \mathrm{MHz}$ Bruker Avance III, and the resulting data were analyzed using MestReNova software, version 10.0.2-15465.

IR measurements. IR measurements were performed on a Bruker Tensor 27 spectrometer equipped with a diamond ATR accessory (32 scans; $4 \mathrm{~cm}^{-1}$ resolution). For the Si NP samples, the dried Si NP powder was firmly pressed onto the ATR crystal; for the pure silanols, a drop was placed on the crystal.

XPS measurements. XPS measurements were performed on a JEOL JPS-9200 photoelectron spectrometer, using a $12 \mathrm{kV}$ and $20 \mathrm{~mA}$ monochromatic $\mathrm{Al} \mathrm{K \alpha}$ source. The analyzer pass energy was $10 \mathrm{eV}$, and the take-off angle between sample and detector was set at $80^{\circ}$. Data was analyzed using the CasaXPS program, version 2.3.18PR1.0. Samples were prepared by drop coating a Si NP solution onto a plasma-cleaned gold surface.

TEM measurements. TEM measurements were performed on a JEOL JEM-1400 plus (WEMC - TEM $120 \mathrm{kV}$ ). Samples were prepared by evaporating a drop of dilute Si NP solution in chloroform onto an ultrathin Carbon Type-A support film with a 400 mesh on top of a copper grid with an approximate hole size of $42 \mu \mathrm{m}$ (TED PELLA, Inc.). Samples were allowed to dry for $30 \mathrm{~min}$ prior to imaging.

High-resolution TEM measurements. High-resolution TEM measurements were performed on a Tecnai G2 F20 Super Twin TEM microscope at $200 \mathrm{kV}$ with a LaB6 emitter. The microscope was equipped with a high-angle annular dark-field (HAADF) detector for scanning transmission electron microscopy (STEM) imaging. STEM imaging and all analytical work was performed with a probe size of $1 \mathrm{~nm}$, resulting in a beam current of about $0.5 \mathrm{nA}$. TEM images were collected using a GATAN US1000 $2 \mathrm{~K}$ HR $200 \mathrm{kV} \mathrm{CCD} \mathrm{camera.} \mathrm{The} \mathrm{CCD} \mathrm{line} \mathrm{traces} \mathrm{were} \mathrm{collected} \mathrm{fully} \mathrm{automatically} \mathrm{using}$ the Tecnai G2 user interface and processed with both Digital Micrograph software Version 2.3 and the Tecnai Imaging and Analysis (TIA) software Version 1.9.162.

Optical properties. Optical properties (in DMSO, unless noted otherwise) were studied using an Edinburgh Instruments FLS900 fluorescence spectrometer (continuous: $450 \mathrm{~W}$ xenon lamp as the excitation source; for lifetime: $444 \mathrm{~nm}$ LED laser) for the fluorescence properties, and a Varian Cary 50 UV-vis spectrophotometer for the absorption measurements (scan rate $600 \mathrm{~nm} \min ^{-1}$; spectra corrected for solvent absorption). Results were fitted with a 3-exponential decay. For the quantum yield, fluorescein in $0.1 \mathrm{M}$ aqueous $\mathrm{NaOH}$ was used as a reference, with an absolute quantum yield of $0.88 \pm 0.03 .{ }^{41}$

\section{Results and discussion}

\section{Silanol-based functionalization of silicon nanoparticles}

The passivation of H-Si NPs with silanol compounds (Fig. 1) proceeded readily at room temperature with a straightforward procedure: silanol was simply added to 
a H-Si NP suspension in DMSO (as obtained without further purification from $\mathrm{Si}$ $\mathrm{NP}$ synthesis), and the mixture was stirred for $1 \mathrm{~h}$ at room temperature. The ease of this reaction is in line with the calculated exothermicity, namely $\Delta H=-16.9 \mathrm{kcal} \mathrm{mol}^{-1}$ in DMSO, as obtained from quantum-chemical wB97XD/ $6-311+G(d, p)$ calculations using a dielectric SMD model mimicking DMSO. The reason for the extra $30 \mathrm{~min}$ of stirring time, compared to the analogous reaction on flat surfaces, ${ }^{37}$ was to ensure a high packing density on the Si NP surface, as this would minimize future oxidation. Escorihuela et al. further reported that for flat surfaces, the reaction speed could be increased by irradiation with UV light. However, this was not tested here, as the subsequent (relatively slow) evaporation of DMSO in vacuo meant the Si NPs would be exposed to silanol for at least $2 \mathrm{~h}$ anyway.

For both silanols $\mathbf{1}$ and 3, excess silanol was removed simultaneously with DMSO, simplifying the purification procedure; only one extraction step with diethyl ether was necessary to remove the remaining traces of unbound silanol and obtain a dry powder. Meanwhile, 2 could not be removed by vacuum at the current temperature. Therefore, for this silanol, three extractions with diethyl ether were performed to obtain a dry powder. In all cases, an estimated yield of $\sim 45 \%$ was obtained, assuming all Si NP surface atoms had reacted with the silanol, and no $\mathrm{Si}-\mathrm{H}$ bonds remained.

To confirm that the passivation process did not affect the size of the Si NPs, TEM imaging was performed. For both passivated and non-passivated Si NPs, the average size of the particles was around $6 \pm 1 \mathrm{~nm}$ (Fig. S13†), slightly $(\sim 1.5 \mathrm{~nm})$ smaller than previously reported by Pujari et al. for H-Si NPs synthesized using similar conditions. ${ }^{34}$

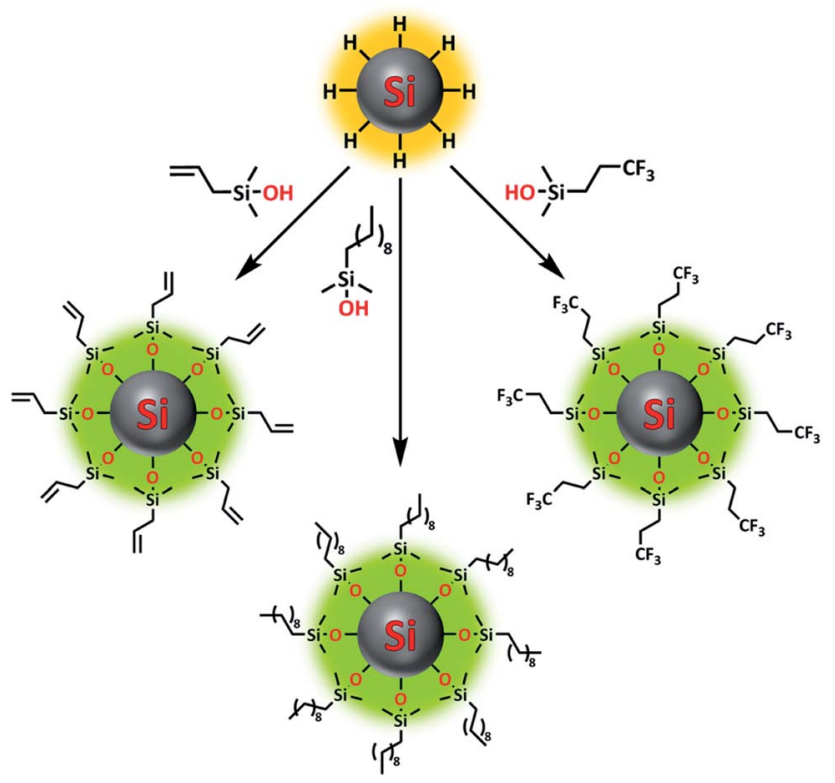

Fig. 1 Overview image showing functionalization of silicon nanoparticles using various silanol compounds. 
High-resolution TEM of single Si NPs confirmed the crystallinity of the functionalized NPs, in line with data shown by Pujari et al. ${ }^{34}$ Additionally, the interplanar spacing of the crystalline particles was measured to be $0.31 \mathrm{~nm}$ in the (111) direction, and $0.19 \mathrm{~nm}$ in the (110) direction (Fig. 2D and E). This corresponds to literature values found for crystalline silicon wafers (3.13 and $1.92 \AA$, respectively). ${ }^{42}$

Recently, a paper by Oliinyk et al. raised the question whether Si NPs could really be formed in a microwave reaction. ${ }^{43}$ Even though they specifically doubted their formation in an aqueous solution, and suggested the formation of fluorescent NPs of a carbon or hybrid composition, their investigation is also relevant here. Based on the interplanar crystal spacing found, and the silicon signal observed using XPS (vide infra), we would argue that crystalline Si NPs were indeed formed in this reaction, rather than hybrid organo-silicon or carbon NPs. Furthermore, Oliinyk et al. attributed the source of fluorescence in the paper by Zhong et al. ${ }^{36}$ to the formation of carbon nanoparticles and/or organic fluorophores. If this had been the case here, the organic fluorophores should have been visible in the NMR and IR spectra discussed below. However, in these spectra, only signals from the silanol coating could be seen, indicating that no other functional groups were present in the sample. Combined, these data imply the formation of well-defined, fluorescent, crystalline Si NPs, rather than non-crystalline and hybrid carbon nanodots, in the microwave reaction described above.

\section{Characterization of silanol-functionalized particles}

NMR spectroscopy. The passivated particles were first characterized using NMR to determine the purity and success of passivation. A representative spectrum of Si NPs passivated with compound 1 (1-Si NPs) is shown in Fig. 3 (for the remaining spectra, see ESI S2 $\uparrow$ ). For clarity, the spectrum of the pure silanol is shown underneath in light grey.

All signals belonging to the alkyl groups of the silanol can clearly be seen in the spectrum of the functionalized Si NPs, while the signal from the -OH group has disappeared (5.55 ppm in DMSO- $\mathrm{d}_{6}$, Fig. S1 $\dagger$ ). This indicates that the silanol has successfully reacted via the $-\mathrm{OH}$ group. In addition, the integration ratio of the peaks closely matches the ratio found for the unbound silanol, although some deviation is sometimes found due to the low signal of the functionalized NPs.

Binding is further confirmed by the broadening of the silanol peaks, caused by attachment of the organic compound to a slowly-rotating NP. ${ }^{19,25}$ Since rotation

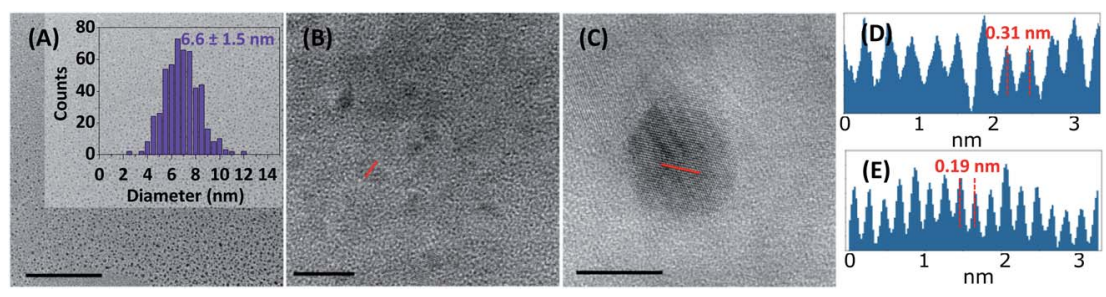

Fig. 2 High-resolution TEM images of 3-Si NPs. Scale bars are $200 \mathrm{~nm}(\mathrm{~A})$ and $10 \mathrm{~nm}$ (B and $C$ ). The insert in (A) shows the size distribution. (D) shows the interplanar spacing of a Si(111) NP measured along the red line in (C), and (E) shows the spacing of a Si(110) NP measured in (B). 


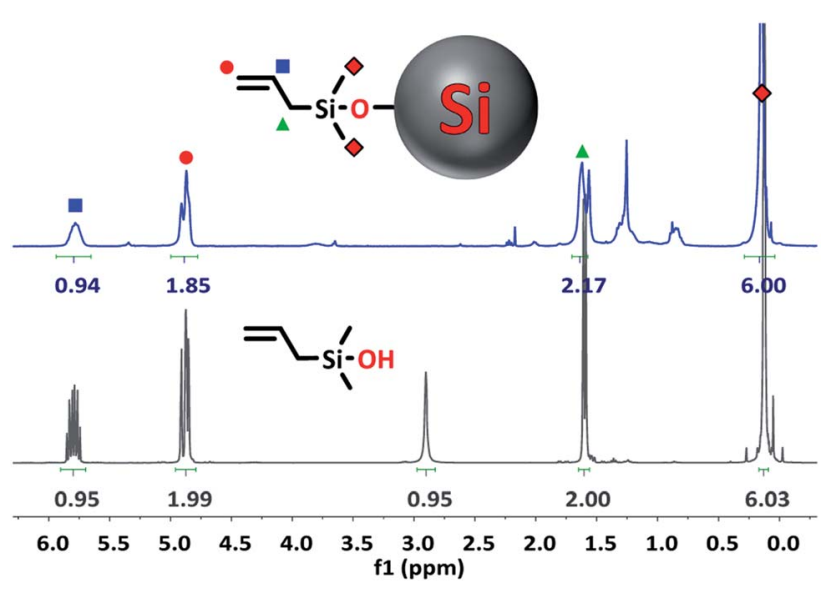

Fig. $3{ }^{1} \mathrm{H}$ NMR spectrum of 1 -Si NPs (top) and pure unbound 1 (bottom) in $\mathrm{CDCl}_{3}$.

speed is related to size, more severe peak broadening is observed for larger particles. This is why the peak broadening here is more severe compared to the spectra of the 1-4 nm NPs reported in the literature. ${ }^{19,25}$ Meanwhile, ${ }^{1} \mathrm{H}$ NMR of $\mathrm{TiO}_{2}$ NPs of $\sim 10.4 \times 7.4 \mathrm{~nm}$ showed spectra with nearly unresolvable peaks. ${ }^{44,45}$

Final proof of successful binding is given by the slight change in chemical shift for hydrogen atoms belonging to the methyl groups of the silanol (Fig. 3, $\sim 0.2$ $\mathrm{ppm}$ ). This shift is upfield when measured in $\mathrm{CDCl}_{3}$, and downfield when measured in DMSO- $\mathrm{d}_{6}$. Hydrogen atoms further up the alkyl chain of the silanol seem to be unaffected by the Si NP surface.

In addition to this, only small contaminant peaks can be found in the spectrum, indicating that the purification was successful. Since NPs, due to their slowtumbling nature, only give very small signals compared to organic compounds, any contaminants are clearly visible in the spectrum.

IR spectroscopy. Since NMR analysis only yielded low signals and broad peaks, ATR-IR was used as extra confirmation of successful silanol attachment. Fig. 4 presents the ATR-IR spectrum of 1-functionalized Si NPs (1-Si NPs), compared to that of the dimerized form of $\mathbf{1}\left(\mathbf{1}_{2}\right)$. Both spectra contain a narrow band at $1257 \mathrm{~cm}^{-1}$, indicative of the presence of $\mathrm{Si}-\mathrm{CH}_{3}$ groups. ${ }^{24,31,46}$ Other characteristic peaks of silanol can also be seen in the 1-Si NP spectrum, such as the $\mathrm{C}=\mathrm{C}$ bond vibrations at 1632, 990, 932, and $893 \mathrm{~cm}^{-1}$, and the $=\mathrm{C}-\mathrm{H}$ vibration at $3080 \mathrm{~cm}^{-1}$. Additionally, the strong band at $1059 \mathrm{~cm}^{-1}$ indicates the presence of a Si-O-Si bond, as expected after passivation, while the absence of a broad peak at $\sim 3270 \mathrm{~cm}^{-1}$ reflects the disappearance of the $-\mathrm{OH}$ group.

For this sample in particular, the IR spectrum is interesting, as it provides further evidence that the silanol has indeed reacted via the $-\mathrm{OH}$ group, rather than the vinyl group. Not only would binding via the vinyl group lead to the disappearance of the $\mathrm{C}=\mathrm{C}$ signals at $1632,990,932$ and $893 \mathrm{~cm}^{-1}$, it would also lead to the formation of a new signal at around $1210 \mathrm{~cm}^{-1} \cdot{ }^{46}$

The absence of this latter peak is not directly clear from the spectrum in Fig. 4. However, when the difference spectrum between passivated and non-passivated Si NPs is plotted, the lack of signal in this region becomes clear (Fig. S5†). In 


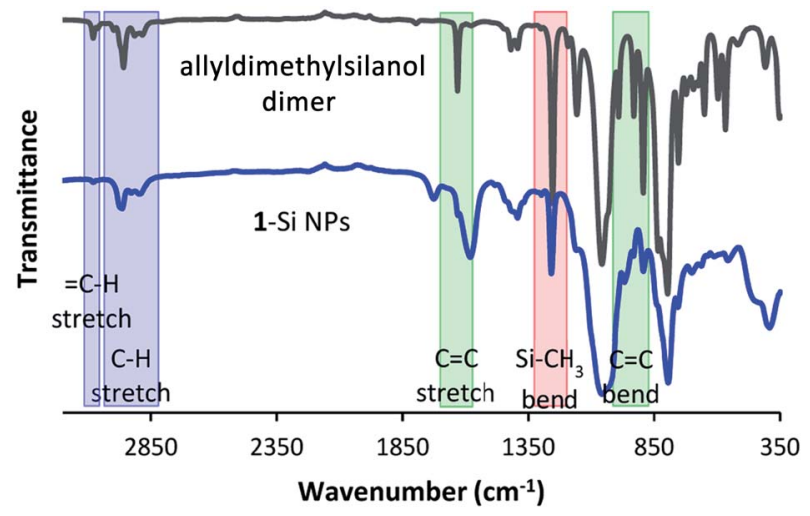

Fig. 4 ATR-IR spectra of $1_{2}$ and 1-Si NPs. Several notable peaks are highlighted.

addition, further signals characteristic of $\mathbf{1}$ also show up in the difference spectrum, including several peaks in the fingerprint region. This is also observed for the other passivations: the difference spectra show characteristic peaks belonging to the silanol used during the reaction (Fig. S6 and S7†). Only for 2-Si NPs is the comparison less clear, due to the small number of characteristic peaks that can be uniquely attributed to 2 .

XPS analysis. To confirm that the IR signal around $1060 \mathrm{~cm}^{-1}$ is indeed due to the binding of the silanol, and not primarily a result of oxidation, XPS analysis was performed. For a well-passivated, non-oxidized sample, a peak $<101 \mathrm{eV}$ is expected in the silicon $2 p$ narrow scan. Meanwhile, for a completely oxidized sample, the peak should be found at $>103 \mathrm{eV} \cdot{ }^{34}$ For the silanol-coated particles, a clear peak was found at $101.8 \mathrm{eV}$, indicating that the Si NPs are partially oxidized (Fig. 5, wide scan in Fig. S8 $\dagger$ ). This is in line with the nature of the silanol functionalization, in which for every silanol ligand attached to the Si NPs, two silicon atoms will be directly bound to an oxygen atom. These silicon atoms need not always be located at the surface of the Si NP, as the oxygen atom can "bury" itself inside the Si NP. As a result, the binding energy of $>2$ silicon atoms can be affected, resulting in an average Si 2p binding energy of $102 \mathrm{eV}$. In addition, a small fraction of oxidation of the initial Si NP core is typically observed under these conditions, which can - in principle - be removed by HF etching. ${ }^{34}$ Since the silanol functionalization itself adds oxygen to the NP, such etching was not performed in this case. This Si $2 p$ binding energy of $101.8 \mathrm{eV}$ was found for all the functionalized Si NPs, with minor $(0.3 \mathrm{eV})$ differences likely due to charging effects in the XPS. ${ }^{47}$

As no peak at $>103 \mathrm{eV}$ is present in Fig. 5, we tentatively attribute the peak at $1060 \mathrm{~cm}^{-1}$, as observed in the IR spectrum, to the $\mathrm{Si}-\mathrm{O}-\mathrm{Si}$ bonds from the silanol functionalization, rather than oxidation by atmospheric oxygen.

Optical properties. Next, the effect of silanol coating on the optical properties of the Si NPs was determined. To this end, the properties were compared to those of H-Si NPs. It was shown that passivation with silanol leads to a $\sim 50 \mathrm{~nm}$ blueshift in both the excitation and emission spectra (Fig. 6). This is likely due to the introduction of oxygen atoms into the Si NPs during silanol coating, which has already been shown to lead to a blue-shift in the spectra. ${ }^{29}$ Interestingly, no shift was observed for 2-Si NPs (Table 1). It is likely that some minor oxidation of 


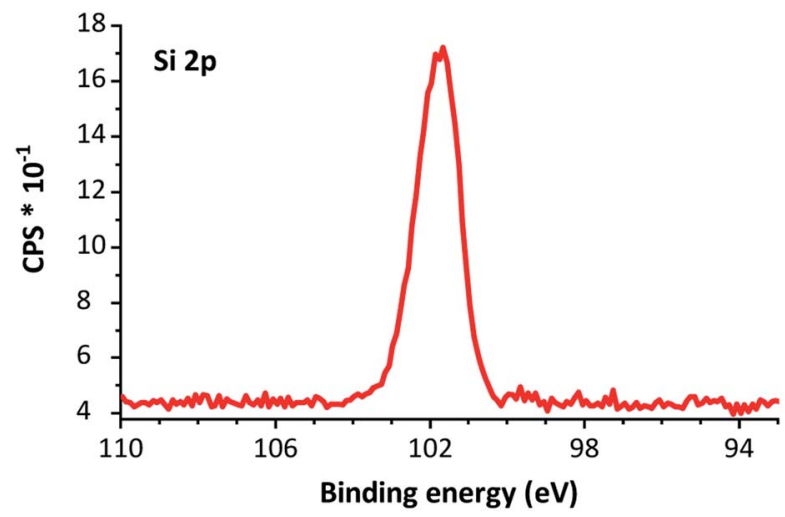

Fig. 5 XPS silicon $2 p$ narrow scan of 2-Si NPs. The peak is centered around $101.8 \mathrm{eV}$.

1- and 3-Si NPs occurred during processing, as the smaller dimethylsilanol ligands, due to their bulkiness, cannot reach $100 \%$ passivation. Meanwhile, the large, hydrophobic alkyl chain of $\mathbf{2}$ is more effective in blocking incoming oxidants, and will therefore offer better protection against oxidation. Indeed, XPS of 3-Si NPs after the measurement of optical properties shows the presence of a small amount of silicon oxide (Fig. S9†).

Besides shifting the positions of the excitation and emission maxima, the silanol coating seems to reduce the spectral width of the Si NPs. For nonpassivated Si NPs, the spectral widths of the excitation and emission spectra were 0.68 and $0.53 \mathrm{eV}$, respectively. For 1-passivated Si NPs the excitation width decreased to $0.57 \mathrm{eV}$, and for 3-Si NPs the excitation and emission width decreased to 0.43 and $0.41 \mathrm{eV}$, respectively. Meanwhile, no change in spectral width was observed for 2-Si NPs; the excitation spectrum had a spectral width of $0.69 \mathrm{eV}$, and the emission spectrum a width of $0.50 \mathrm{eV}$.

Finally, the fluorescence lifetime and quantum yield were also affected by the passivation process. The average fluorescence lifetime of 3-Si NPs increased from 4.1 to $6.9 \mathrm{~ns}$ after passivation, while for 2-Si NPs the lifetime decreased to $3.3 \mathrm{~ns}$.

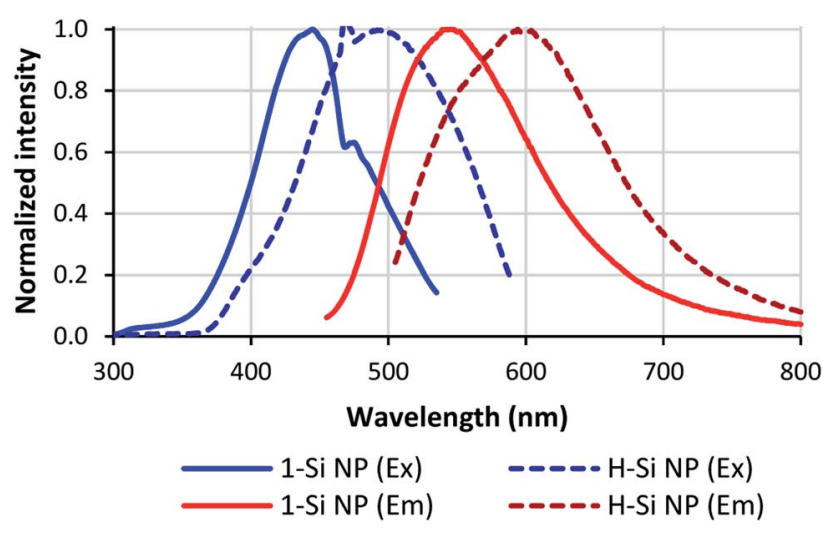

Fig. 6 Optical properties of Si NPs. Full lines: Si NPs passivated with 1; dashed lines: nonpassivated Si NPs. Excitation spectra are shown in blue, emission spectra in red. 
Table 1 Optical properties of silanol-coated Si NPs

\begin{tabular}{lllll}
\hline Sample & $\begin{array}{l}\lambda_{\mathrm{ex}} \\
\max (\mathrm{nm})\end{array}$ & $\begin{array}{l}\lambda_{\mathrm{em}} \\
\max (\mathrm{nm})\end{array}$ & $\begin{array}{l}\text { Average lifetime } \\
(\mathrm{ns})\end{array}$ & Quantum yield \\
\hline H-Si NP & 496 & 594 & 4.1 & $16 \%^{a}$ \\
1-Si NP & 445 & 545 & 4.2 & $30 \%^{a}$ \\
2-Si NP & 495 & 589 & 3.3 & $20 \%^{a}$ \\
3-Si NP & 443 & 539 & 6.9 & $27 \%^{a}$ \\
Thioglucose Si NPs & 370 & 460 & - & $35 \%^{b}$ \\
${ }^{a}$ QY w.r.t. fluorescein in $0.1 \mathrm{M} \mathrm{NaOH}^{b}{ }^{b}$ QY w.r.t. quinine sulfate.
\end{tabular}

These short ns lifetimes are typical for Si NPs with alkyl termination, prepared using wet-chemical methods, as shown in previous studies on free-standing $\mathrm{Si}$ NPs. ${ }^{31,48-52}$ In addition, it has been shown that an electronegative cap (such as silanol) or an electronegative environment (such as DMSO) can also cause short ns lifetimes for Si NPs. ${ }^{53}$

The quantum yield of the Si NPs increased for all silanols, though the increase for 2-Si NPs was not as large as for the other silanols. This increase could be partially due to the purification procedure for the passivated Si NPs; as the H-Si NP sample was not purified before quantum yield determination (to minimize oxidation), this sample contains traces of non-fluorescent impurities that increase the optical absorption of the sample, and thereby slightly decrease the apparent quantum yield for the H-Si NPs compared to the purified samples. The quantum yield for these silanol-functionalized Si NPs compares favorably with other modes of functionalization. ${ }^{34}$

\section{Reactivity of silanol-based Si NPs: water-soluble, glucose-coated Si NPs}

As a proof of concept, to show that water-soluble, bio-functional Si NPs could be prepared in this manner, 1-thio- $\beta$-D-glucose tetraacetate was reacted with 1-Si NPs via a thiol-ene click reaction (Fig. 7 and 8). Previous research on thiol-ene click reactions used a reaction time of $1 \mathrm{~h} .{ }^{47}$ Here, due to the size and bulkiness of the sugar ligand, a longer reaction time of $3.5 \mathrm{~h}$ was used, to ensure a higher packing density. Due to the extensive purification needed during synthesis of these NPs, and

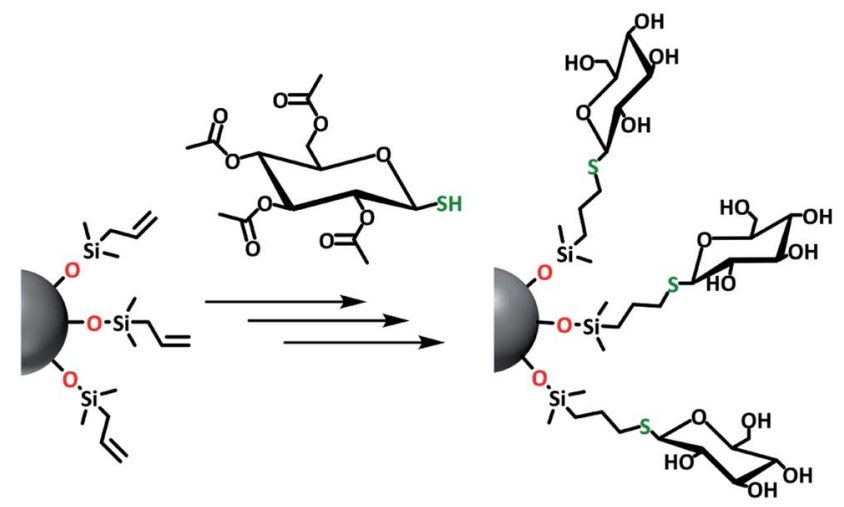

Fig. 7 Schematic overview of the synthesis of water-soluble, glucose-coated Si NPs. 

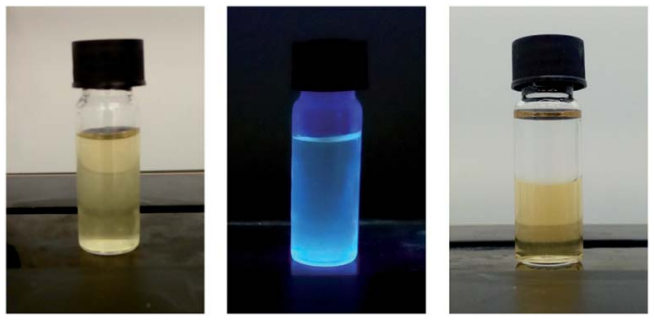

Fig. 8 Glucose-coated Si NPs in water under normal light (left) and $365 \mathrm{~nm}$ irradiation (middle). The right picture shows the solubility of glucose-coated Si NPs in hexane (top layer) and water (bottom layer).

the small scale of the experiment ( $30 \mathrm{mg}$ of H-Si NPs), this reaction yielded only $27 \mathrm{mg}$. Successful binding of the protected thiol-sugar was confirmed by IR, with the disappearance of the S-H stretch vibration after the click reaction (Fig. S15†).

After deprotection, the thioglucose-coated Si NPs were dispersed in water for fluorescence measurements. An excitation maximum of $370 \mathrm{~nm}$ and an emission maximum of $460 \mathrm{~nm}$ were found, with a quantum yield of $35 \%$ compared to quinine sulfate (Fig. S12†). This is a significant blue-shift compared to the Si NPs coated only with silanol, likely due to UV-induced oxidation.

\section{Conclusions}

We have demonstrated a fast, easy and efficient way to passivate hydrogenterminated $\mathrm{Si}$ nanoparticles (H-Si NPs) using silanol compounds at room temperature. The reaction was specific enough to allow the presence of other functional groups in the silanol ligand, and both the passivation and purification of the Si NPs were simple procedures with opportunities for scale-up. The silanolcoated Si NPs produced here showed little oxidation and improved quantum yields. Finally, purified silanol-coated Si NPs could be produced and purified from hydrogen-terminated Si NPs in under $4 \mathrm{~h}$, showing a significant improvement over previous methods for H-Si NP functionalization.

\section{Conflicts of interest}

There are no conflicts to declare.

\section{Acknowledgements}

The authors acknowledge funding from the Netherlands Organization for Scientific Research (NWO) in the framework of the Materials for Sustainability program. Low-resolution electron microscopy work was performed at the Wageningen Electron Microscopy Center (WEMC) of Wageningen University. Barend van Lagen is acknowledged for helpful instrumental assistance.

\section{Notes and references}

1 X. Cheng, S. B. Lowe, P. J. Reece and J. J. Gooding, Chem. Soc. Rev., 2014, 43, 2680 . 
2 Y. Su, X. Ji and Y. He, Adv. Mater., 2016, 28, 10567.

3 S. Wu, Y. Zhong, Y. Zhou, B. Song, B. Chu, X. Ji, Y. Wu, Y. Su and Y. He, J. Am. Chem. Soc., 2015, 137, 14726.

4 B. Ghosh, Y. Masuda, Y. Wakayama, Y. Imanaka, J. Inoue, K. Hashi, K. Deguchi, H. Yamada, Y. Sakka, S. Ohki, et al., Adv. Funct. Mater., 2014, 24, 7151.

5 S. Coe, W.-K. Woo, M. Bawendi and V. Bulovic, Nature, 2002, 420, 800.

6 L. Yang, Y. Liu, Y.-L. Zhong, X.-X. Jiang, B. Song, X.-Y. Ji, Y.-Y. Su, L.-S. Liao and Y. He, Appl. Phys. Lett., 2015, 106, 173109.

7 I. Robel, V. Subramanian, M. Kuno and P. V. Kamat, J. Am. Chem. Soc., 2006, 128, 2385.

8 G. Y. Kwak, T. G. Kim, S. Hong, A. Kim, M. H. Ha and K. J. Kim, Sol. Energy, 2018, 164, 89.

9 B. Liu, J. Hu, L. Jia, J. Liu, X. Ren, X. Zhang, X. Guo and S. Liu, Sol. Energy, 2018, 167, 102.

10 T. Subramani, J. Chen, Y. L. Sun, W. Jevasuwan and N. Fukata, Nano Energy, 2017, 35, 154.

11 J. Duan, H. Zhang, Q. Tang, B. He and L. Yu, J. Mater. Chem. A, 2015, 3, 17497.

12 B. Song and Y. He, Nano Today, 2019, 26, 149.

13 S. Bhattacharjee, I. M. C. M. Rietjens, M. P. Singh, T. M. Atkins, T. K. Purkait, Z. Xu, S. Regli, A. Shukaliak, R. J. Clark, B. S. Mitchell, et al., Nanoscale, 2013, 5, 4870.

14 L. Ruizendaal, S. Bhattacharjee, K. Pournazari, M. Rosso-Vasic, L. H. J. De Haan, G. M. Alink, A. T. M. Marcelis and H. Zuilhof, Nanotoxicology, 2009, 3, 339.

15 X. Li, Y. He, S. S. Talukdar and M. T. Swihart, Langmuir, 2003, 19, 8490.

16 F. Erogbogbo, K. T. Yong, R. Hu, W. C. Law, H. Ding, C. W. Chang, P. N. Prasad and M. T. Swihart, ACS Nano, 2010, 4, 5131.

17 W. Ren, Y. Wang, Q. Tan, J. Yu, U. J. Etim, Z. Zhong and F. Su, Electrochim. Acta, 2019, 320, 134625.

18 F. Erogbogbo, C. A. Tien, C. W. Chang, K. T. Yong, W. C. Law, H. Ding, I. Roy, M. T. Swihart and P. N. Prasad, Bioconjugate Chem., 2011, 22, 1081.

19 A. Shiohara, S. Hanada, S. Prabakar, K. Fujioka, T. H. Lim, K. Yamamoto, P. T. Northcote and R. D. Tilley, J. Am. Chem. Soc., 2010, 132, 248.

20 M. Dasog, G. B. De los Reyes, L. V. Titova, F. A. Hegmann and J. G. C. Veinot, ACS Nano, 2014, 8, 9636.

21 C. M. Hessel, E. J. Henderson and J. G. C. Veinot, Chem. Mater., 2006, 18, 6139.

22 Z. Kang, Y. Liu, C. H. A. Tsang, D. D. D. Ma, X. Fan, N.-B. Wong and S.-T. Lee, Adv. Mater., 2009, 21, 661.

23 M. Rosso-vasic, E. Spruijt, Z. Popovíc, K. Overgaag, B. van Lagen, B. Grandidier, D. Vanmaekelbergh, D. Domínguez-Gutiérrez, L. De Cola and H. Zuilhof, J. Mater. Chem., 2009, 19, 5926.

24 C.-S. Yang, R. A. Bley, S. M. Kauzlarich, H. W. H. Lee and G. R. Delgado, J. Am. Chem. Soc., 1999, 121, 5191.

25 W. Biesta, B. van Lagen, V. S. Gevaert, A. T. M. Marcelis, J. M. J. Paulusse, M. W. F. Nielen and H. Zuilhof, Chem. Mater., 2012, 24, 4311.

26 Y. Yu, C. M. Hessel, T. D. Bogart, M. G. Panthani, M. R. Rasch and B. A. Korgel, Langmuir, 2013, 29, 1533. 
27 J. A. Kelly, A. M. Shukaliak, M. D. Fleischauer and J. G. C. Veinot, J. Am. Chem. Soc., 2011, 133, 9564.

28 M. Dasog and J. G. C. Veinot, Phys. Status Solidi A, 2012, 209, 1844.

29 Y. Yu, C. E. Rowland, R. D. Schaller and B. A. Korgel, Langmuir, 2015, 31, 6886.

30 M. Dasog, K. Bader and J. G. C. Veinot, Chem. Mater., 2015, 27, 1153.

31 M. Rosso-vasic, E. Spruijt, B. Van Lagen, L. De Cola and H. Zuilhof, Small, 2008, 4, 1835.

32 I. M. D. Höhlein, J. Kehrle, T. K. Purkait, J. G. C. Veinot and B. Rieger, Nanoscale, 2015, 7, 914.

33 J. M. Buriak, Chem. Mater., 2014, 26, 763.

34 S. P. Pujari, H. Driss, F. Bannani, B. van Lagen and H. Zuilhof, Chem. Mater., 2018, 30, 6503.

35 Y. Zhong, X. Sun, S. Wang, F. Peng, F. Bao, Y. Su, Y. Li, S.-T. Lee and Y. He, ACS Nano, 2015, 9, 5958.

36 Y. Zhong, F. Peng, F. Bao, S. Wang, X. Ji, L. Yang, Y. Su, S.-T. Lee and Y. He, J. Am. Chem. Soc., 2013, 135, 8350.

37 J. Escorihuela and H. Zuilhof, J. Am. Chem. Soc., 2017, 139, 5870.

38 N. S. Bhairamadgi, S. Gangarapu, M. A. Caipa Campos, J. M. J. Paulusse, C. J. M. Van Rijn and H. Zuilhof, Langmuir, 2013, 29, 4535.

39 M. A. C. Campos, J. M. J. Paulusse and H. Zuilhof, Chem. Commun., 2010, 46, 5512.

40 K. Ágoston, A. Dobó, J. Rákó, J. Kerékgyárto and Z. Szurmai, Carbohydr. Res., 2001, 330, 183.

41 K. Suzuki, A. Kobayashi, S. Kaneko, K. Takehira, T. Yoshihara, H. Ishida, Y. Shiina, S. Oishi and S. Tobita, Phys. Chem. Chem. Phys., 2009, 11, 9850.

42 C. Xiao, J. Guo, P. Zhang, C. Chen, L. Chen and L. Qian, Sci. Rep., 2017, 7, 40750.

43 B. V. Oliinyk, D. Korytko, V. Lysenko and S. Alekseev, Chem. Mater., 2019, 31, 7167.

44 E. Schechtel, R. Dören, H. Frerichs, M. Panthöfer, M. Mondeshki and W. Tremel, Langmuir, 2019, 35, 12518.

45 Z. Hens and J. C. Martins, Chem. Mater., 2013, 25, 1211.

46 B. Arkles and G. Larson, Silicon Compounds: Silanes \& Silicones, Gelest Inc., Morrisville, 3rd edn, 2013.

47 L. Ruizendaal, S. P. Pujari, V. Gevaerts, J. M. J. Paulusse and H. Zuilhof, Chem.Asian J., 2011, 6, 2776.

48 K. Dohnalová, A. N. Poddubny, A. A. Prokofiev, W. D. De Boer, C. P. Umesh, J. M. Paulusse, H. Zuilhof and T. Gregorkiewicz, Light: Sci. Appl., 2013, 2, e47.

49 K. Dohnalová, A. Fučíková, C. P. Umesh, J. Humpolíčková, J. M. J. Paulusse, J. Valenta, H. Zuilhof, M. Hof and T. Gregorkiewicz, Small, 2012, 8, 3185.

50 D. S. English, L. E. Pell, Z. Yu, P. F. Barbara and B. A. Korgel, Nano Lett., 2002, 2, 681.

51 K. Kůsova, O. Cibulka, K. Dohnalová, I. Pelant, J. Valenta, A. Fučíková, K. Žídek, J. Lang, J. Englich, P. Matějka, et al., ACS Nano, 2010, 4, 4495.

52 J. H. Warner, A. Hoshino, K. Yamamoto and R. D. Tilley, Angew. Chem., Int. Ed., 2005, 44, 4550.

53 A. N. Poddubny and K. Dohnalová, Phys. Rev. B, 2014, 90, 245439. 